\title{
TRPV1 is a Responding Channel for Acupuncture Manipulation in Mice Peripheral and Central Nerve System
}

\author{
Hsiao-Chun Chen ${ }^{\mathrm{a}} \quad$ Ming-Yen Chen ${ }^{\mathrm{a}} \quad$ Ching-Liang Hsieha, ${ }^{\mathrm{a}, \mathrm{b}} \mathrm{c} \quad$ Shu-Yih Wu ${ }^{\mathrm{d}}$ \\ Hsin-Cheng Hsue Yi-Wen Lin ${ }^{a, f}$
}

${ }^{a}$ College of Chinese Medicine, Graduate Institute of Acupuncture Science, China Medical University, Taichung, ${ }^{b}$ College of Chinese Medicine, Graduate Institute of Integrated Medicine, China Medical University, Taichung, 'China Medical University Hospital, Department of Chinese Medicine, Taichung, ${ }^{\mathrm{d}}$ Department of Rehabilitation Medicine, Mackay Memorial Hospital, Taipei, ${ }^{\mathrm{e} C o l l e g e}$ of Chinese Medicine, School of Post-Baccalaureate Chinese Medicine, China Medical University, Taichung, ${ }^{f}$ Chinese Medicine Research Center, China Medical University, Taichung, Taiwan

\author{
Key Words \\ Acupuncture $\cdot$ Dorsal root ganglion - Spinal cord $・$ TRPV1 $・$ PERK $・$ PNR1
}

\begin{abstract}
Background/Aims: Acupuncture involves inserting a fine needle into a specific point, often called an acupoint, thereby initiating a therapeutic effect accompanied by phenomena such as soreness, heaviness, fullness, and numbness. Acupoints are characterized as points located in deep tissues with abundant sensory nerve terminals, which suggests that there is a strong relationship between acupoints and peripheral sensory afferents. In this study, we determined whether manual acupuncture (MA) or different frequencies of electroacupuncture (EA) share similar mechanisms for activating excitatory neurotransmission. Methods: We performed MA or EA at acupoint ST36 and we also used western blot and immunostaining techniques to determine neural changes at the peripheral dorsal root ganglion (DRG), spinal cord (SC), and somatosensory cortex (SSC) levels. Results: Our results show that either MA or EA at the ST36 acupoint significantly increased components of the TRPV1-related signaling pathway, such as pPKA, pPI3K, pPKC-pERK, and pAKT (but not pp38 or pJNK) at the peripheral DRG and central SC-SSC levels. Furthermore, excitatory phosphorylated $\mathrm{N}$-methyl-D-aspartate receptor (pNMDA) and pCaMKII (but not pNR2B, pCaMKII $\delta$, or pCaMKII $\gamma$ ) also increased. These molecules could not increase in the DRG and SC-SSC of TRPV1-/-mice. Conclusion: Our data demonstrates that both MA and EA can activate excitatory signals in either peripheral or central levels. We also define that TRPV1 is crucial for an acupuncture effect and then initiate excitatory pNR1-pCaMKII pathway, at peripheral DRG and central SC-SSC level. We suggest that the TRPV1 signaling pathway is highly correlated to Acupuncture effect that implies the real clinical significance.




\section{Cellular Physiology Cell Physiol Biochem 2018;49:1813-1824

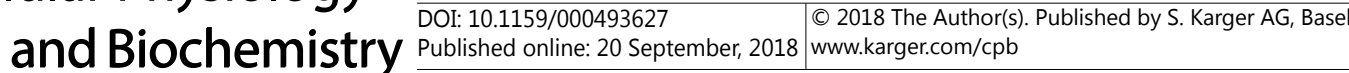 \\ Chen et al.: TRPV1 as Responding Channel for Acupuncture}

\section{Introduction}

Acupuncture is a major part of traditional Chinese medicine that has been used for more than 3000 years and its therapeutic effect is accepted worldwide. The acupuncture procedure is performed by inserting a needle into acupoints manually (manual acupuncture, MA) or with electric enhancement (electroacupuncture, EA). It has been reported that acupoints are highly abundant at peripheral sensory nerve endings and manipulating acupoints could change the signals from the transient receptor potential vanilloid receptor 1 (TRPV1) and c-fos in local tissue and the spinal cord (SC) dorsal horn [1, 2]. Goldman et al. used microdialysis and high performance liquid chromatography to show that adenosine triphosphate (ATP), which is degraded into adenosine to elicit its analgesic effect, was released at acupoints during MA [3]. Acupuncture has been used to control body weight [4], reduce epilepsy [5], ameliorate learning and memory impairment [6], and for pain management [7-10]. The analgesic effect of acupuncture activates the release of endogenous opiates [11] and adenosine [3]. Acupuncture involves inserting a fine needle into a specific point, which is often called an acupoint, thereby initiating a phenomenon known as de-qi. De-qi is a crucial indicator during acupuncture treatments, which includes obvious feelings such as soreness, heaviness, fullness, and numbness. Acupoints are characterized as points located in deep tissues with abundant sensory nerve terminals, thereby suggesting a strong relationship between acupoints and peripheral sensory afferents. One study demonstrated that EA significantly increases the expression of TRPV1 to that observed in the control body weight group [4].

TRPV1, a capsaicin receptor, was first described as a thermosensitive channel isolated from sensory neurons mainly in the dorsal root ganglion (DRG) [12]. Members of the TRPV family contain six transmembrane segments with six ankyrin repeats and large $\mathrm{N}$ and C-termini in the cytoplasm. TRPV1 has high $\mathrm{Ca}^{2+}$ permeability and can be activated by capsaicin, heat $\left(>43^{\circ} \mathrm{C}\right)$, and exposure to low $\mathrm{pH}$. Acupuncture can trigger local ATP release and calcium wave propagation through active TRPV receptors [13-16]. TRPV1 may also be an acupuncture-responding channel because it is highly expressed in acupoints with abundant sensory nerve endings that deliver signals to the SC $[1,2]$.

$\mathrm{N}$-methyl-D-aspartate (NMDA) receptors are a target of glutamate, which is an excitatory neurotransmitter in the mammalian peripheral and central nervous system. The four types of NRs comprise AMPA, NMDA, kainite, and metabotropic receptors $[17,18]$. NRs are heteromeric protein complexes, which comprise a $\mathrm{Ca}^{2+}$-permeable ion channel with four subunits. There are three main NDMAR families: NR1, NR2, and NR3. The NR1, NR2A, NR2B, and NR2D subunits are distributed in the DRG and SC $[19,20]$. Activation of NRs can induce the influx of $\mathrm{Ca}^{2+}$ and then lead to the activation of $\mathrm{Ca}^{2+} /$ calmodulin-dependent protein kinase 2 (CaMKII) for signaling in the postsynaptic density [21]. There are four isomers of CaMKII in mammals, i.e., $\alpha, \beta, \gamma$, and $\delta$. CaMKII is a holoenzyme and a serine/threonine kinase that is highly expressed in the brain mainly participates in its phosphorylation [22]. Jang et al. reported that peripheral release of glutamate induced by local nerve stimulation leads to tactile hypersensitivity of the skin. In addition, the PKA- and PKC-dependent mechanisms mediated mainly by activating NMDA are also induced [23]. Wong et al. demonstrated that injecting nerve growth factor into the masseter muscle initiates mechanical sensitization in rats. The duration of mechanical sensitization was associated with increased peripheral activation of NMDA by masseter muscle afferent fibers [24].

In this study, we applied MA or different frequencies of EA at the ST36 acupoint to identify neural activation by acupuncture. Our rational is that either MA or EA delivers mechanical stimulation to activate TRPV1 and NMDA at the ST36 acupoint and passes peripheral signals to the central nervous system. We used immunostaining, western blot, and genetic techniques to verify the involvement of TRPV1 and associated signaling pathways after acupuncture treatment. Our results showed that both MA and different frequencies of EA could activate the TRPV1 and excitatory pNR1-pCaMKII $\alpha$-pERK signaling pathway in the DRG, SC, and somatosensory cortex (SSC) areas. The levels of these compounds were not 


\section{Cellular Physiology Cell Physiol Biochem 2018;49:1813-1824

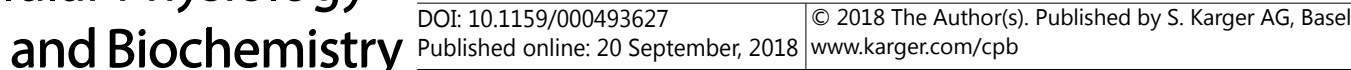 \\ Chen et al.: TRPV1 as Responding Channel for Acupuncture}

increased in TRPV1/-mice, which suggests that TRPV1 is a key mechanosensitive channel in the mammalian peripheral DRG and central SC-SSC pathway.

\section{Materials and Methods}

\section{Animals}

Experiments were conducted using male C57/B6 mice (ages 8 to 12 weeks) purchased from BioLASCO Co. Ltd (Taipei, Taiwan) and used as a backcross pool for all lines of genetically modified mutant mice. Knockout mice of TRPV1 was obtained from the Jackson Laboratory (Bar Harbor, ME). Mice were randomly assigned to five groups ( $\mathrm{n}=8$ per group): (1) Control, (2) MA, (3) $2 \mathrm{~Hz}$ EA, (4) $15 \mathrm{~Hz}$ EA, and (5) $50 \mathrm{~Hz}$ EA. The sample size required for an alpha of 0.05 and a power of $80 \%$ was eight animals per group. After arrival, the mice were housed under a 12/12 h light/dark cycle, where water and food were available ad libitum. All of the procedures were approved by the Institute of Animal Care and Use Committee of China Medical University (permit No. 2016-061) and they were conducted in accordance with the Guide for the use of Laboratory Animals provided by the National Research Council and the ethical guidelines of the International Association for the Study of Pain. The number of animals used and their suffering were minimized.

\section{Acupuncture manipulation}

Acupuncture was delivered using stainless steel needles (0.5 inch, 32 G, YU KUANG, Taiwan), which were inserted into the muscle layer at a depth of $2-3 \mathrm{~mm}$ in the bilateral ST36 acupoint, the location of ST36 is approximately 3-4 mm below and 1-2 mm lateral to the midpoint of the knee in mice, under $1 \%$ isoflurane anesthetization. In the control group, a needle was inserted into the ST36 acupoint without any rotation or twisting. To ensure an insertion depth of $3 \mathrm{~mm}$, a piece of tape was attached to the needle, which only left sufficient space for manipulation and the needle tip of $3 \mathrm{~mm}$. In the MA group, the tape was used as a guide and the needle was twisted anticlockwise by $360^{\circ}$, and then back for one twist at a speed of approximately 1 turn/s. The protocol used two twists every $3 \mathrm{~min}$ for a duration of $15 \mathrm{~min}$. In the EA group, we applied 2, 15, and $50 \mathrm{~Hz}$ EA by delivering electrical stimulation with a Trio 300 electrical stimulator (Grand Medical Instrument Co. Ltd). A stimulator (Trio 300, Ito, Japan) delivered 100- $\mu$ s square pulses of 1 $\mathrm{mA}$ for $15 \mathrm{~min}$ at 2,15 , and $50 \mathrm{~Hz}$.

Tissue sampling and western blot analysis

Bilateral L3-L5 DRG, L3-L5 SC dorsal horn, and SSC neurons were excised immediately to extract proteins. The total proteins were prepared by homogenizing the DRG, SC, and SSC in lysis buffer containing 50 mM Tris-HCl (pH 7.4), 250 mM NaCl, 1\% NP-40, 5 mM EDTA, 50 mM NaF, 1 mM Na3VO4, 0.02\% NaNO3, and $1 \times$ protease inhibitor cocktail (AMRESCO). The extracted proteins (30 $\mu \mathrm{g}$ per sample according to the BCA protein assay) were subjected to $8 \%$ SDS-Tris glycine gel electrophoresis and transferred to a PVDF membrane. The membrane was blocked with 5\% non-fat milk in TBS-T buffer (10 mM Tris pH 7.5, 100 $\mathrm{mM} \mathrm{NaCl}, 0.1 \%$ Tween 20 ), incubated with the first antibody in TBS-T and $1 \%$ bovine serum albumin, and incubated for $1 \mathrm{~h}$ at room temperature. A peroxidase-conjugated anti-rabbit antibody (1:5000) was used as the secondary antibody. The bands were visualized using an enhanced chemiluminescencent substrate kit (PIERCE) with LAS-3000 Fujifilm (Fuji Photo Film Co. Ltd). If appropriate, the image intensities of specific bands were quantified with NIH ImageJ software (Bethesda, MD, USA). The protein ratios were obtained by dividing the target protein intensities by the intensity of $\alpha$-tubulin in the same sample. The calculated ratios were then adjusted by dividing the ratios from the same comparison group relative to the control.

\section{Immunohistochemical staining}

Mice were anesthetized with isoflurane and then perfused transcardially with 4\% paraformaldehyde. Next, we dissected the SC from the lumbar section. The tissue samples were embedded in paraffin. The paraffin-embedded sections were cut to a thickness of $5 \mu \mathrm{m}$ and pasted onto micro-slide glasses coated with APS. The sections were post-fixed briefly with $4 \%$ paraformaldehyde for $3 \mathrm{~min}$ and then incubated with blocking solution containing 3\% BSA, $0.1 \%$ Triton X-100, and $0.02 \%$ sodium azide in PBS for $2 \mathrm{~h}$ at room temperature. After blocking, the sections were incubated at $4{ }^{\circ} \mathrm{C}$ overnight with the primary antibodies against anti-pNR1 (1:1000, Cell Signalling, Danvers, USA, ref. 3381), anti-pNR2B (1:1000, Cell Signalling, 


\section{Cellular Physiology Cell Physiol Biochem 2018;49:1813-1824 and BiOChemistry \begin{tabular}{l|l} 
DOI: 10.1159/000493627 & $\begin{array}{l}\text { (c) } 2018 \text { The Author(s). Published by S. Karger AG, Basel } \\
\text { www.karger.com/cpb }\end{array}$
\end{tabular} \\ Chen et al.: TRPV1 as Responding Channel for Acupuncture}

ref. 4208), anti-pPKA (1:1000, Novus, Littleton USA, ref. NBP1-40676), pPI3K (1:1000, Millipore, ref. 4228), anti-pPKC (1:1000, Cell Signalling, ref. 2060), anti-pAkt (1:1000, Millipore, ref. 4060), anti-phosphorylated $\mathrm{Ca}^{2+} /$ calmodulin-dependent PK (pCaMKII $\alpha$ 1:1000, Cell Signalling, ref. 11945), anti-CaMKII $\delta$ (1:1000, Abcam, Cambridge, Massachusetts, USA, ref. ab54927), anti-CaMKII $(1: 1000$, Abcam, ref. ab37999), antipERK (1:1000, Novus, ref. NB100-92406), anti-pp38 (1:1000, Novus, ref. NBP1-51458), anti-pJNK (1:1000, Novus, ref AF1205). The secondary antibody was goat anti-rabbit (1:500) antibody (Molecular Probes, Carlsbad, CA, USA). We incubated the slices with avidin-biotin horseradish peroxidase complex $(1 \mathrm{~h})$, washed them three times with $0.1 \mathrm{M}$ Tris buffer ( $5 \mathrm{~min}$ each), and then developed them in diaminobenzidine tetrahydrochloride (1-2 min), before washing three times with $0.1 \mathrm{M}$ Tris buffer ( $5 \mathrm{~min}$ each). Finally, the sections were incubated with $0.1 \mathrm{M}$ Tris buffer to stop the reaction. The slides were mounted with cover slips and visualized by using a CKX41 microscope with an Olympus U-RFLT50 Power Supply Unit (Olympus, Tokyo, Japan).

\section{Statistical analysis}

All of the data were expressed as the mean \pm standard error. Significant differences between the control group, MA group, and 2, 15, and $50 \mathrm{~Hz}$ EA groups were tested using ANOVA, followed by a post hoc Tukey's test. $\mathrm{p}<0.05$ was considered significantly different.

\section{Results}

MA and EA increased immunopositive signals for the TRPV1-associated signaling pathway in the peripheral DRG

We performed Western blot analysis of DRGs in mice from groups subjected to MA or different frequencies of EA. Our results indicated that the pPKA protein expression levels were higher in MA (Fig. $1 \mathrm{~A}, \mathrm{p}<0.05, \mathrm{n}=6$ ). The same results were also obtained in the 2 , 15 , and $50 \mathrm{~Hz}$ EA groups (Fig. $1 \mathrm{~A}$, all $\mathrm{p}<0.05, \mathrm{n}=6$ ). We then verified that $\mathrm{pPI} 3 \mathrm{~K}$ was also augmented after MA manipulation (Fig. $1 \mathrm{~A}$, all $\mathrm{p}<0.05, \mathrm{n}=6$ ). Similar results were also obtained for the pPKC and pAKT (Fig. 1A, all $\mathrm{p}<0.05, \mathrm{n}=6$ ). We found that the pERK levels increased after MA and EA stimulation (Fig. 1B, all $p<0.05, n=6$ ). However, similar results were not obtained for the pp38 (Fig. $1 \mathrm{~B}$, all $\mathrm{p}>0.05, \mathrm{n}=6$ ) and pJNK pathways (Fig. 1B, all $\mathrm{p}>0.05, \mathrm{n}=6$ ).

We showed that control mice DRGs exhibited normal pNR1 expression, whereas the pNR1 protein expression levels were higher in the MA group (Fig. $1 B, p<0.05, n=6$ ). Similar results were also obtained for the EA groups, where the $2 \mathrm{~Hz}$ EA, $15 \mathrm{~Hz}$ EA, and 50 $\mathrm{Hz}$ EA groups all had higher pNR1 expression levels compared with the control group (Fig. $1 \mathrm{~B}$, all $\mathrm{p}<0.05, \mathrm{n}=6$ ). However, the pNR2B levels did not differ between the control and experimental groups in the mice DRGs, as shown in Fig. 1C. In the MA group, the pCaMKII $\alpha$ protein expression levels were higher than those of the control mice (Fig. $1 \mathrm{C}, \mathrm{p}<0.05, \mathrm{n}=6$ ). Similarly, in the 2,15, and $50 \mathrm{~Hz}$ groups, EA dramatically increased the pCaMKII $\alpha$ expression levels (Fig. $1 \mathrm{C}$, all $\mathrm{p}<0.05, \mathrm{n}=6$ ). However, the expression levels of pCaMKII $\delta$ were the same in all of the groups (Fig. $1 \mathrm{C}$, all $p>0.05, n=6$ ). Similar results were also obtained for the DRGs incubated with pCaMKII $\gamma$ antibody (Fig. 1C, all $\mathrm{p}>0.05, \mathrm{n}=6$ ).

\section{MA and EA activate the TRPV1-associated signaling pathway in the central SC level}

Next, we examined TRPV1-associated mechanisms in the central SC level. We found that the pPKA protein expression levels were higher in the MA and EA groups (Fig. $2 \mathrm{~A}$, all $\mathrm{p}<0.05$, $n=6$ ). We then found that the pPI3K levels were increased in the MA group (Fig. $2 \mathrm{~A}$, all $\mathrm{p}<$ $0.05, \mathrm{n}=6$ ) and similar data were obtained for the pPKC levels (Fig. $2 \mathrm{~A}$, all $\mathrm{p}<0.05, \mathrm{n}=6$ ). Moreover, the pAKT (Fig. 2A, all $\mathrm{p}<0.05, \mathrm{n}=6$ ) and pERK levels were increased after MA and EA stimulation (Fig. $2 B$, all $\mathrm{p}<0.05, \mathrm{n}=6$ ). However, similar patterns were not detected in the pp38 (Fig. 2B, all p > 0.05, $n=6$ ) and pJNK pathways (Fig. 2B, all $p>0.05, n=6$ ).

We found that MA and EA significantly potentiated the expression of pNR1 protein in the SC of mice (Fig. 2B, all $\mathrm{p}<0.05, \mathrm{n}=6$ ). We found that the levels of pNR2B did not differ 


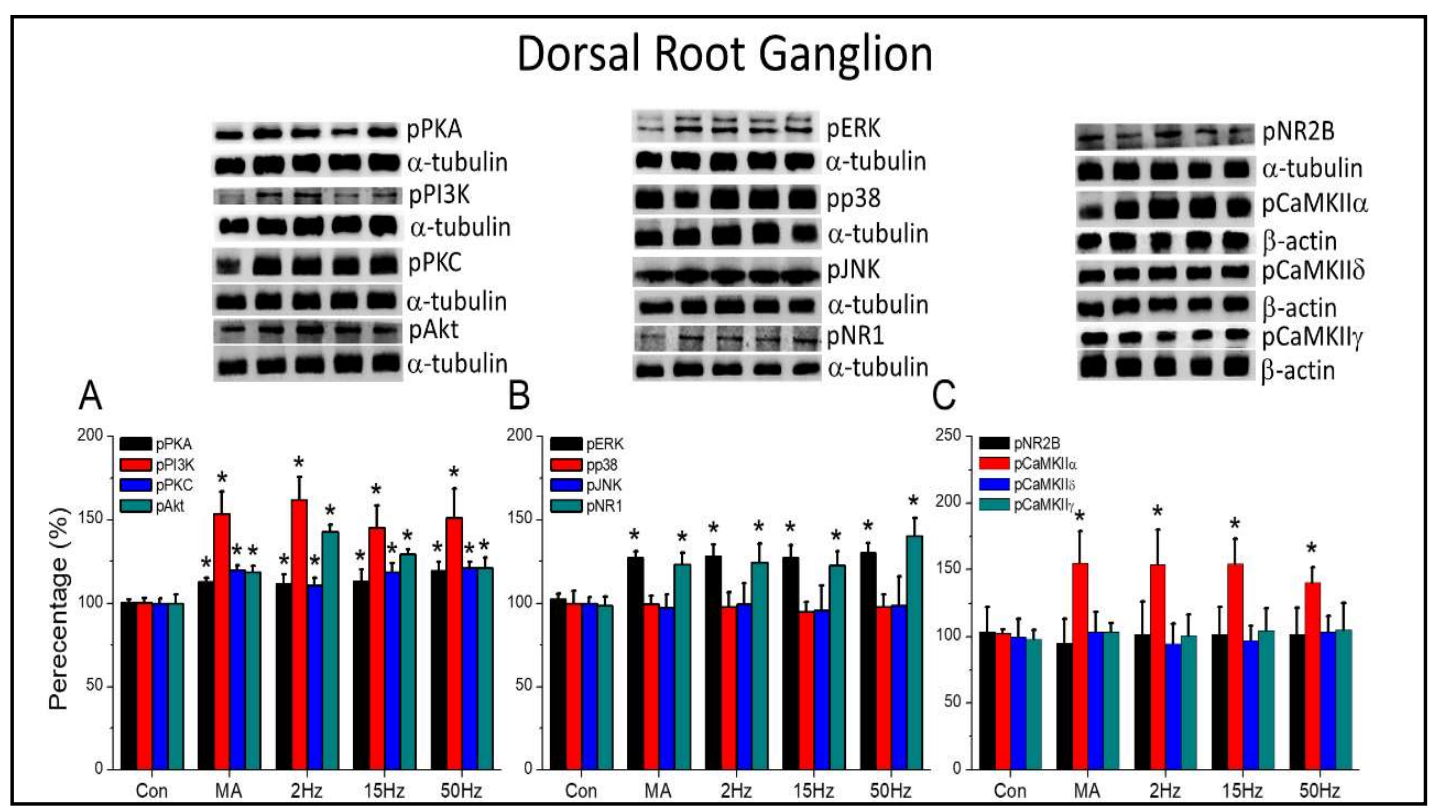

Fig. 1. Expression levels of TRPV1-associated signaling pathway proteins in DRG after MA and EA manipulation. (A) pPKA, pPI3K, pPKC, and pAKT, (B) pERK, pp38, pJNK, and pNR1 (C) pNR2B, pCaMKII $\alpha$, pCaMKII $\delta$, and pCaMKII $\gamma$ expression levels in tissues from the Con, MA, $2 \mathrm{~Hz}, 15 \mathrm{~Hz}$, and $50 \mathrm{~Hz}$ EA groups (from left to right). Con = Control; MA = Manual acupuncture; EA = Electroacupuncture. ${ }^{*} \mathrm{p}<0.05$ compared with the control group. The western blot bands at the top show the target protein. The lower bands are internal controls ( $\beta$-actin or $\alpha$-tubulin).

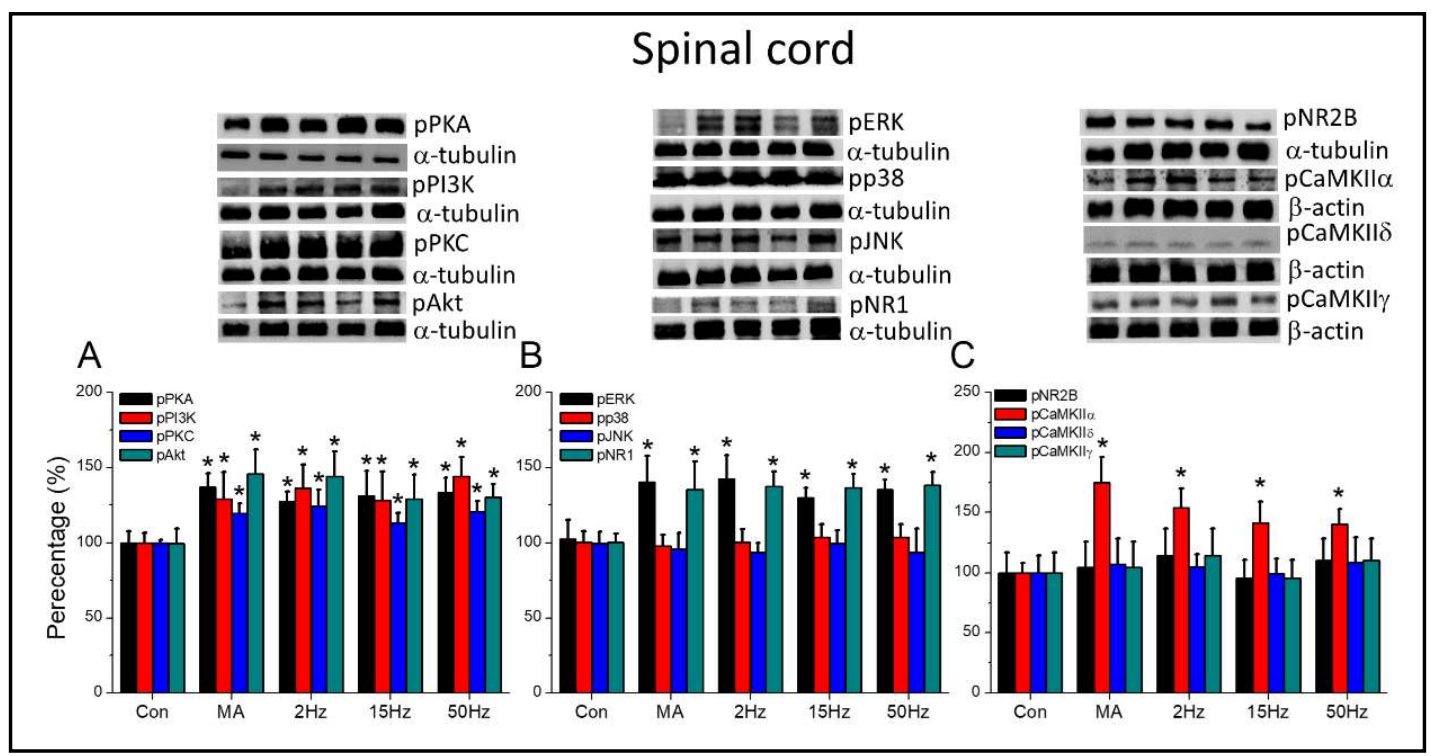

Fig. 2. Expression levels of TRPV1-associated signaling pathway proteins in the lumbar SC after MA and EA manipulation. (A) pPKA, pPI3K, pPKC, and pAKT, (B) pERK, pp38, pJNK, and pNR1 (C) pNR2B, pCaMKII $\alpha$, pCaMKII $\delta$, and pCaMKII $\gamma$ expression levels in tissues from the Con, MA, $2 \mathrm{~Hz}, 15 \mathrm{~Hz}$, and $50 \mathrm{~Hz}$ EA groups (from left to right). $\mathrm{Con}=$ Control; $\mathrm{MA}=$ Manual acupuncture; $\mathrm{EA}=$ Electroacupuncture. ${ }^{*} \mathrm{p}<0.05$ compared with the control group. The western blot bands at the top show the target protein. The lower bands are internal controls ( $\beta$-actin or $\alpha$-tubulin).

among all of the groups (Fig. 2C, all $\mathrm{p}>0.05, \mathrm{n}=6$ ). We then showed that the pCaMKII $\alpha$ levels were higher in the MA group than the control (Fig. $2 \mathrm{C}, \mathrm{p}<0.05, \mathrm{n}=6$ ). In the 2, 15, and $50 \mathrm{~Hz}$ groups, EA potentiated the expression of pCaMKII $\alpha$ (Fig. $2 \mathrm{C}, \mathrm{p}<0.05$ ). However, the levels of KARGER 


\section{Cellular Physiology Cell Physiol Biochem 2018;49:1813-1824 and Biochemistry \begin{tabular}{l|l} 
DOI: 10.1159/000493627 & $\begin{array}{l}\text { (c) } 2018 \text { The Author(s). Published by S. Karger AG, Basel } \\
\text { Published }\end{array}$
\end{tabular}

pCaMKII $\delta$ and pCaMKII $\gamma$ were not changed by either MA or different EA frequencies of in the SC of mice (Fig. 2C, all $\mathrm{p}>0.05, \mathrm{n}=6$ ).

\section{MA and EA activate the TRPV1-associated signaling pathway in the brain SSC level}

We next found that the pPKA protein expression levels were higher in SSC of both MA and EA groups (Fig. 3A, all $\mathrm{p}<0.05, \mathrm{n}=6$ ). We then found that the pPI3K levels were increased in the MA group (Fig. 3A, all $\mathrm{p}<0.05, \mathrm{n}=6$ ) and similar data were obtained for the pPKC levels (Fig. 3A, $n=6$ ). The pAKT (Fig. 3A, all $p<0.05, n=6$ ) and pERK levels were increased after MA and EA stimulation (Fig. 3B, all $p<0.05, n=6$ ). However, similar patterns were not detected in the pp38 (Fig. 3B, all $p>0.05, n=6$ ) and pJNK pathways (Fig. 3B, all $p>0.05, n$ $=6$ ). We next found that MA significantly potentiated the expression of pNR1 protein in the SSC of mice (Fig. 3B, $\mathrm{p}<0.05, \mathrm{n}=6$ ). In the 2,15 , and $50 \mathrm{~Hz}$ groups, EA also led to higher pNR1 expression levels in the SSC compared with the control group (Fig. 3B, all $p<0.05, n=$ 6). pNR2B did not differ among all of the groups (Fig. $3 \mathrm{C}$, all $\mathrm{p}>0.05, \mathrm{n}=6$ ). The pCaMKII $\alpha$ levels were higher in the MA and EA group (Fig. 3C, $p<0.05, n=6$ ). However, the levels of pCaMKII $\delta$ and pCaMKII $\gamma$ were not changed by either MA or different EA frequencies of in the SSC of mice (Fig. 3C, all $\mathrm{p}>0.05, \mathrm{n}=6$ ).

\section{MA- and EA-induced activation of the TRPV1-related signaling pathway was abolished in}

TRPV1 $1^{-/}$mice in the peripheral DRG

We deleted the TRPV1 gene to determine whether the MA- or EA-induced signaling pathway was abolished in the peripheral DRG level. We found that the pPKA protein expression levels were not significantly different after MA and EA manipulation (Fig. 4A, $\mathrm{p}>0.05, \mathrm{n}=6$ ). Our results suggested that the pPI3K levels were not increased after MA manipulation in TRPV1/-mice DRG (Fig. 4A, all $\mathrm{p}>0.05, \mathrm{n}=6$ ) and similar results were also obtained for the pPKC (Fig. 4A, all $\mathrm{p}>0.05, \mathrm{n}=6$ ) and pAKT levels (Fig. 4A, all $\mathrm{p}>0.05, \mathrm{n}=$ 6). The levels of pERK were not increased after MA and EA stimulation (Fig. 4B, all $p>0.05$, $\mathrm{n}=6$ ), and similar results were obtained for pp38 (Fig. 4B, all $\mathrm{p}>0.05, \mathrm{n}=6$ ) and pJNK (Fig. $4 \mathrm{~B}$, all $\mathrm{p}>0.05, \mathrm{n}=6$ ).

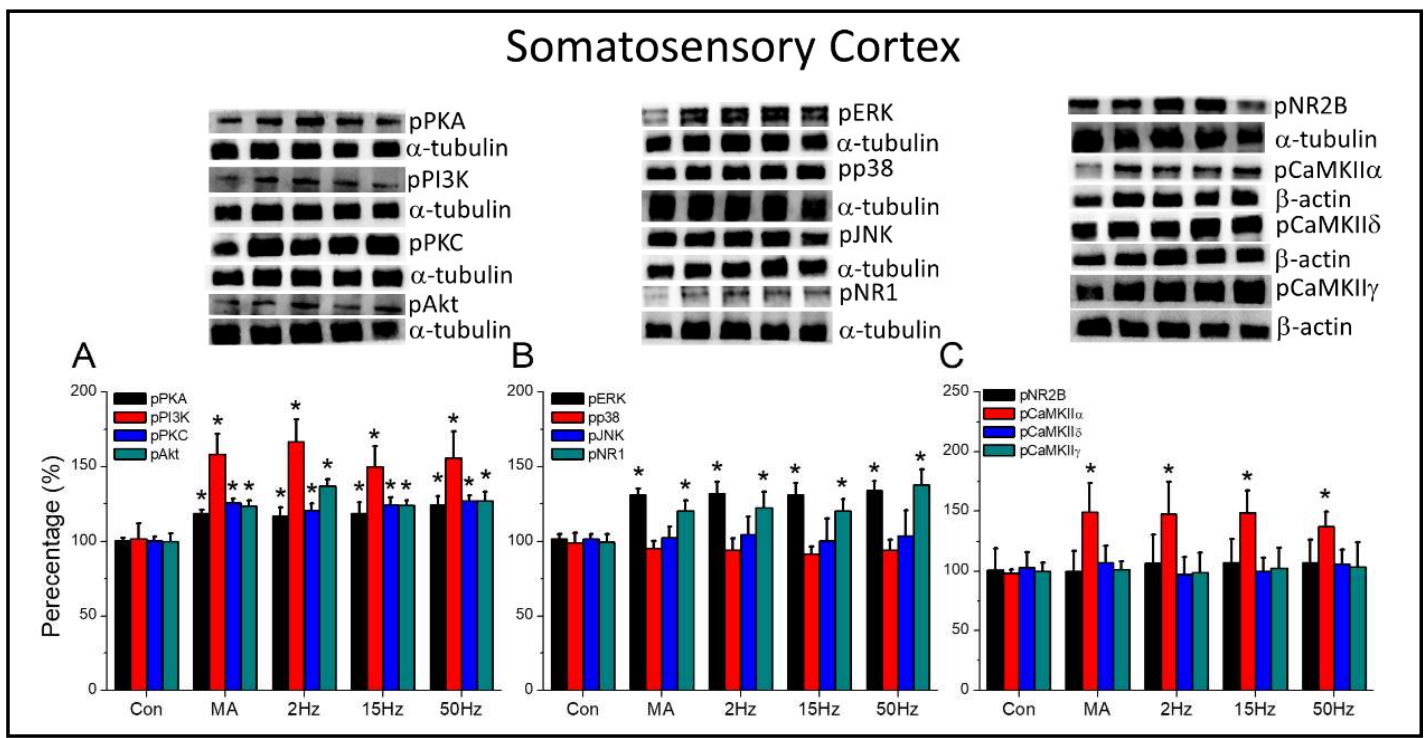

Fig. 3. Expression levels of TRPV1-associated signaling pathway proteins in the SSC after MA and EA manipulation. (A) pPKA, pPI3K, pPKC, and pAKT, (B) pERK, pp38, pJNK, and pNR1 (C) pNR2B, pCaMKII $\alpha$, pCaMKII $\delta$, and pCaMKII $\gamma$ expression levels in tissues from the Con, MA, $2 \mathrm{~Hz}, 15 \mathrm{~Hz}$, and $50 \mathrm{~Hz}$ EA groups (from left to right). Con = Control; MA = Manual acupuncture; $\mathrm{EA}=$ Electroacupuncture. ${ }^{*} \mathrm{p}<0.05$ compared with the control group. The western blot bands at the top show the target protein. The lower bands are internal controls ( $\beta$-actin or $\alpha$-tubulin). 


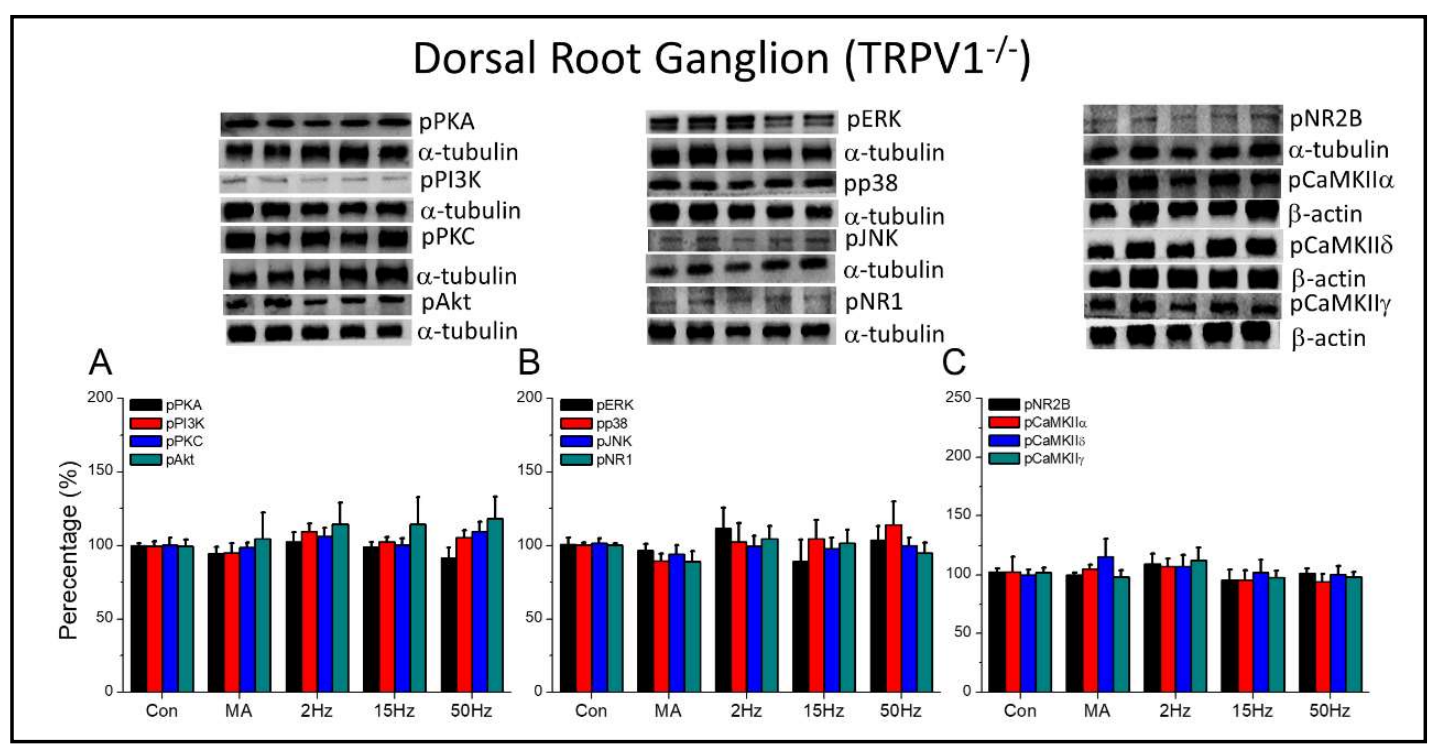

Fig. 4. Expression levels of TRPV1-associated signaling pathway proteins in the DRG from TRPV1-/-mice after MA and EA manipulation. (A) pPKA, pPI3K, pPKC, and pAKT, (B) pERK, pp38, pJNK, and pNR1 (C) pNR2B, pCaMKII $\alpha$, pCaMKII $\delta$, and pCaMKII $\gamma$ expression levels in tissues from the Con, MA, $2 \mathrm{~Hz}, 15 \mathrm{~Hz}$, and $50 \mathrm{~Hz}$ EA groups (from left to right). Con = Control; MA = Manual acupuncture; EA = Electroacupuncture. ${ }^{*} \mathrm{p}<0.05$ compared with the control group. The western blot bands at the top show the target protein. The lower bands are internal controls ( $\beta$-actin or $\alpha$-tubulin).

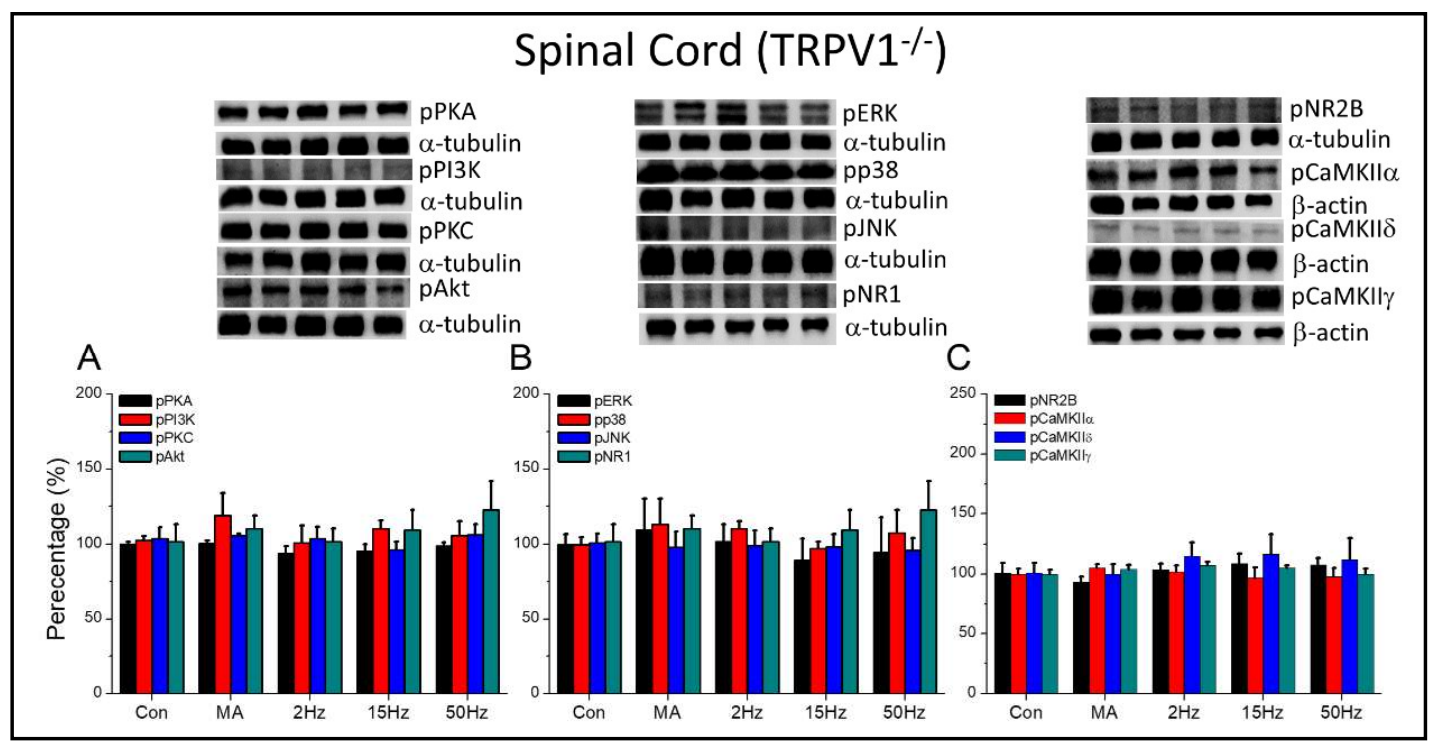

Fig. 5. Expression levels of TRPV1-associated signaling pathway proteins in the lumbar SC from TRPV1-/mice after MA and EA manipulation. (A) pPKA, pPI3K, pPKC, and pAKT, (B) pERK, pp38, pJNK, and pNR1 (C) pNR2B, pCaMKII $\alpha$, pCaMKII $\delta$, and pCaMKII $\gamma$ expression levels in tissues from the Con, MA, $2 \mathrm{~Hz}, 15 \mathrm{~Hz}$, and $50 \mathrm{~Hz}$ EA groups (from left to right). Con = Control; MA = Manual acupuncture; EA = Electroacupuncture. ${ }^{*} \mathrm{p}<0.05$ compared with the control group. The western blot bands at the top show the target protein. The lower bands are internal controls ( $\beta$-actin or $\alpha$-tubulin).

In MA group of TRPV1/-mice, the pNR1 protein expression levels in the DRG were similar to those in the control group (Fig. $4 \mathrm{~B}, \mathrm{p}>0.05, \mathrm{n}=6$ ). Similar results were also obtained for the EA groups (Fig. $4 \mathrm{~B}$, all $\mathrm{p}>0.05, \mathrm{n}=6$ ). The pNR2B levels did not differ among groups (Fig. $4 \mathrm{C}$, all $\mathrm{p}>0.05, \mathrm{n}=6$ ). We also tested whether the pCaMKII levels differed in TRPV1 ${ }^{-1-}$ mice

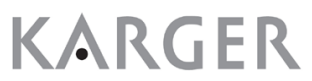


after MA or EA stimulation. The pCaMKII $\alpha$ protein expression levels were not changed in the DRG in any of the groups of TRPV1/-mice (Fig. 4C, $p>0.05, n=6$ ). The pCaMKII $\delta$ levels were similar in all groups (Fig. $4 \mathrm{C}$, all $\mathrm{p}>0.05, \mathrm{n}=6$ ). Moreover, the same results were also obtained for pCaMKII $\gamma$ (Fig. 4C, all $\mathrm{p}>0.05, \mathrm{n}=6$ ).

MA- and EA-induced increases in TRPV1-associated compounds were reduced in the central SC of TRPV1 ${ }^{-1}$ mice

We found that the pPKA protein expression levels were not increased in the SC of the MA and EA manipulation groups (Fig. 5A, p >0.05, $n=6$ ). We showed that the pPI3K levels were not increased after MA or EA manipulation in the SC of TRPV1/-mice (Fig. 5A, all $p>0.05, n$ $=6$ ). Similar results were also obtained for the pPKC (Fig. $5 \mathrm{~A}$, all $\mathrm{p}>0.05, \mathrm{n}=6$ ) and pAKT (Fig. 5A, all $p>0.05, n=6$ ). Our results suggested that the $p E R K$ levels were not increased after MA and EA stimulation (Fig. $5 \mathrm{~B}$, all $\mathrm{p}>0.05, \mathrm{n}=6$ ), and similar results were obtained for pp38 (Fig. 5B, all p > 0.05, $n=6$ ) and pJNK (Fig. 5B, all $p>0.05, n=6$ ).

In TRPV1 $1^{-1-}$ mice SC, the pNR1 protein levels were similar to those in the control group after MA and EA manipulation (Fig. 5B, $\mathrm{p}>0.05, \mathrm{n}=6$ ). The pNR2B levels did not differ among any groups (Fig. $5 \mathrm{C}$, all $\mathrm{p}>0.05, \mathrm{n}=6$ ). The pCaMKII $\alpha$ protein expression levels did not change in the SC among all the groups of TRPV1-/-mice (Fig. $5 C, p>0.05, n=6$ ). The $\mathrm{pCaMKII} \delta$ protein levels were similar in all groups (Fig. $5 \mathrm{C}$, all $\mathrm{p}>0.05, \mathrm{n}=6$ ). There is also not different in pCaMKII $\gamma$ (Fig. 5C, all p > 0.05, $\mathrm{n}=6$ ).

MA- and EA-induced increases in TRPV1-associated compounds were reduced in the central SSC of TRPV1-1-mice

We found that the pPKA protein expression levels were not increased in the SSC of the MA and EA manipulation groups (Fig. $6 \mathrm{~A}$, all $\mathrm{p}>0.05, \mathrm{n}=6$ ). We showed that the pPI3K levels were not increased after MA or EA manipulation in the SC of TRPV1 ${ }^{-/}$mice (Fig. 6A, all $p>0.05, n=6$ ). Similar results were also obtained for the pPKC (Fig. 6A, all $p>0.05, n=6$ ) and pAKT (Fig. 6A, all $\mathrm{p}>0.05, \mathrm{n}=6$ ). Our results suggested that the $\mathrm{pERK}$ levels were not increased after MA and EA stimulation (Fig. 6B, all $p>0.05, n=6$ ), and similar results were

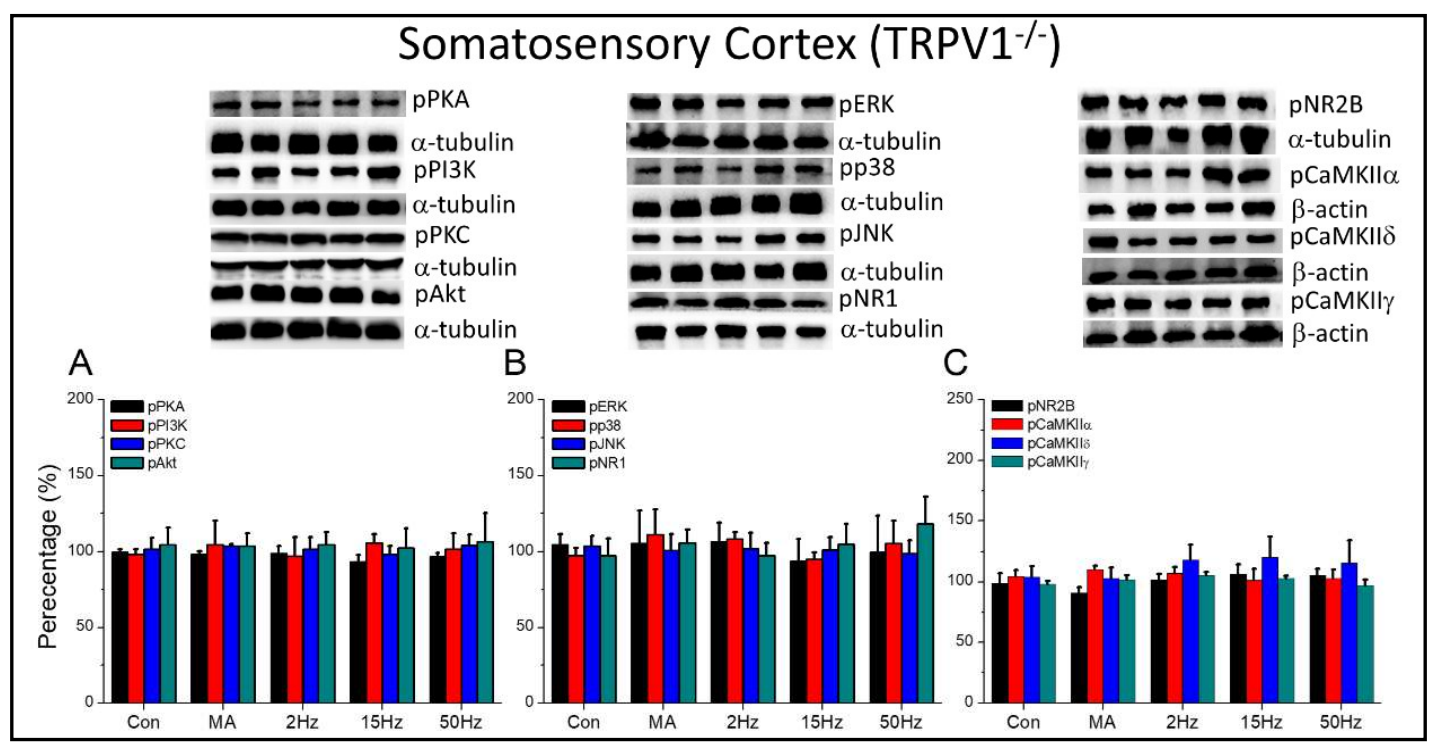

Fig. 6. Expression levels of TRPV1-associated signaling pathway proteins in the SSC from TRPV1-/-mice after MA and EA manipulation. (A) pPKA, pPI3K, pPKC, and pAKT, (B) pERK, pp38, pJNK, and pNR1 (C) pNR2B, pCaMKII $\alpha$, pCaMKII $\delta$, and pCaMKII $\gamma$ expression levels in tissues from the Con, MA, $2 \mathrm{~Hz}, 15 \mathrm{~Hz}$, and $50 \mathrm{~Hz}$ EA groups (from left to right). Con = Control; MA = Manual acupuncture; EA = Electroacupuncture. ${ }^{*} \mathrm{p}<0.05$ compared with the control group. The western blot bands at the top show the target protein. The lower bands are internal controls ( $\beta$-actin or $\alpha$-tubulin). 


\section{Cellular Physiology Cell Physiol Biochem 2018;49:1813-1824 and Biochemistry DOI: 10.1159/000493627 2018 (0) 2018 The Author(s). Published by S. Karger AG, Basel

Fig. 7. Effects of MA or $2 \mathrm{~Hz}$ EA on pPKC- and pCaMKII $\alpha$-positive cells in the SC of mice. Positive cells (brown) in the SC increased after MA or 2 $\mathrm{Hz}$ EA manipulation. (A) pPKC-positive signals in the SC dorsal horn of Con mice. (B) pPKC-positive signals in the SC dorsal

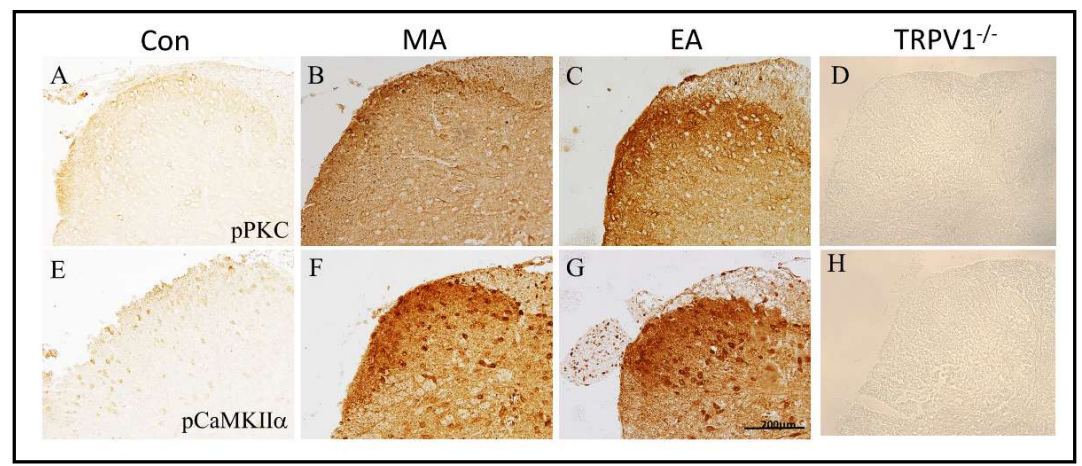
horn of MA mice. (C) pPKC-positive signals in the SC dorsal horn of EA mice. (D) pPKC-positive signals in the SC dorsal horn of TRPV1 $\%$ mice. (E) pCaMKII $\alpha$-positive signals in the SC dorsal horn of Con mice. (F) pCaMKII $\alpha$-positive signals in the SC dorsal horn of MA mice. (G) pCaMKII $\alpha$-positive signals in SC dorsal horn of EA mice. (H) pCaMKII $\alpha$-positive signals in the SC dorsal horn of TRPV1 $\%$ mice.

Fig. 8. Schematic illustration of underlying mechanisms in acupuncture manipulation. Summary diagram of how TRPV1 is crucial for sensing acupuncture and related mechanisms. Our results show that TRPV1 acts as a receptor in acupuncture manipulation. Activation of TRPV1 increases the expression of pPKA, pPI3K, pPKC. Furthermore, pERK and pAKT were also increased. Moreover, aforementioned molecules could be attenuated in TRPV1 $1 \%$ mice.

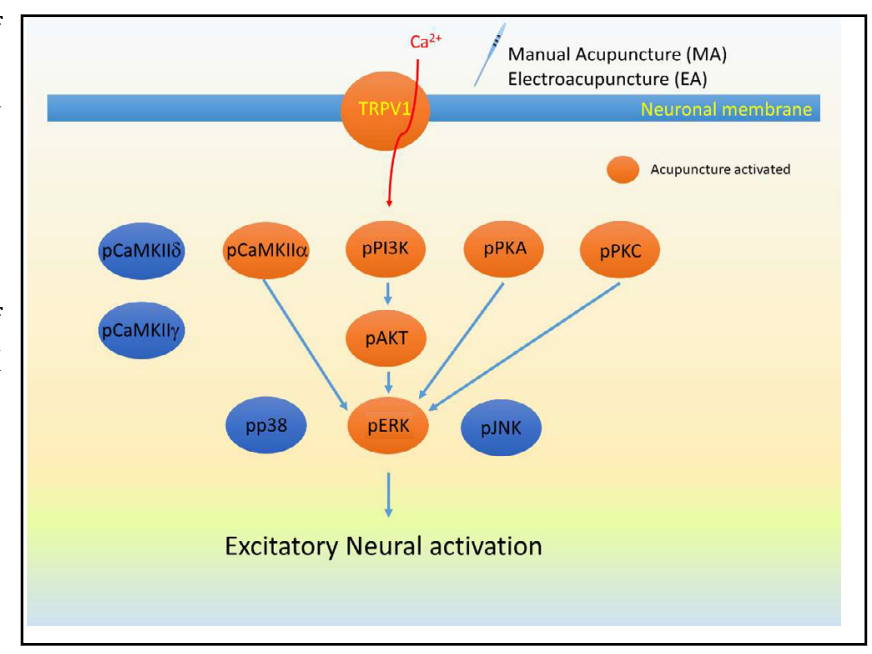

obtained for pp38 (Fig. 6B, all p > 0.05, n =6) and pJNK (Fig. 6B, all p > 0.05, n =6).

In TRPV1/-mice SC, the pNR1 protein levels were similar to those in the control group after MA and EA manipulation (Fig. 6B, all $p>0.05, n=6$ ). The pNR2B levels did not differ among any groups (Fig. $6 \mathrm{C}$, all $\mathrm{p}>0.05, \mathrm{n}=6$ ). The pCaMKII $\alpha$ protein expression levels did not change in the SC among all the groups of TRPV1-/-mice (Fig. $6 \mathrm{C}, \mathrm{p}>0.05, \mathrm{n}=6$ ). The $\mathrm{pCaMKII} \delta$ protein levels were similar in all groups (Fig. $6 \mathrm{C}$, all $\mathrm{p}>0.05, \mathrm{n}=6$ ). There is also not different in pCaMKII $\gamma$ (Fig. 6C, all p > 0.05, $\mathrm{n}=6$ ).

Fig. 7 shows the pPKC and pCaMKII $\alpha$ expression levels in the central SC. Consistent with the western blotting results, the number of pPKC-immunopositive neurons was increased by either MA or EA but not in TRPV1\% mice (Fig. 7A-D). Similarly, the number of pCaMKII $\alpha$ immunopositive neurons was enhanced by MA or EA but not in TRPV1\% mice (Fig. 7E-H). The current study provides molecular mechanisms of acupuncture manipulation on TRPV1 and relevant molecules (Fig. 8).

\section{Discussion}

In the current study, our results suggest that MA or EA at the ST36 acupoint can activate excitatory neural signals in the peripheral DRG and central SC-SSC levels. Our findings also indicate that the levels of TRPV1-associated downstream compounds, such as pPKA, pPI3K, pPKC-pERK, and pAKT, were increased by MA or EA manipulation. Furthermore, excitatory KARGER 
pNR1 and pCaMKII $\alpha$ were increased in the DRG of mice after MA or EA manipulation. These signals were also increased in the central SC-SSC level of mice. Immunohistochemical analyses show that either MA or EA increased neural signals in both the dorsal horn of SC. Our findings provide crucial evidence of neural activation, mainly by activating TRPV1 to induce the pNR1-pCaMKII $\alpha$ signaling pathway in the peripheral DRG and central SC-SSC using MA or EA.

Acupuncture has been reported as effective for pain control by increasing adenosine release $[25,26]$. Previous studies showed that the TRPV1 and pERK protein levels were increased after EA stimulation. Thus, EA can alter the expression of NR1 and TRPV1 in hippocampal CA1 areas in middle cerebral artery occlusion rats [27]. In addition, EA can inhibit long-term potentiation in the SC dorsal horn of rats after nerve injury [28]. We found that pNR1 but not pNR2B was significantly activated by both MA and different frequencies of EA. EA is effective for treating inflammatory pain using ipsilateral and contralateral acupoints, thereby suggesting central activation [9]. Our results indicate that MA and EA could reliably increase neural signals in the peripheral DRG and SC dorsal horn.

Ferrini et al. demonstrated similar IPSPs in lamina II of the spinal dorsal horn in mice via TRPV1 [29], where they used capsaicin (a TRPV1 agonist) to stimulate primary afferent terminal fibers active to release substance P. Subsequently, substance P could induce IPSCs in lamina II (substantia gelatinosa) neurons to produce $\gamma$-amino butyric acid A (GABAA) and glycine. GABA and glycine are mainly inhibitory mediators in the SC [30, 31]. A recent study also demonstrated that the expression of TRPV1 in peripheral nerve fibers was significantly increased after EA stimulation of acupoints but not non-acupoint meridians or non-meridian control skin [1]. Significantly higher expression levels of TRPV1 were found with nNOS in the subepidermal nerve fibers in the acupoints, which increased after EA. This indicates that TRPV1 upregulation after EA is crucial for mediating the transduction of EA signals to the CNS [1].

However, the activation of TRPV1 in primary afferent fibers can increase the release of glutamate in postsynaptic regions [32]. Excitatory-postsynaptic currents in the dorsal horn neurons of mice are observed after glutamate release due to capsaicin stimulation. Recently, Ryu et al. found that EA can reduce pain by decreasing the phosphorylation of NMDA [33]. In addition, Jang et al. found that intrathecal injection of dizocilpine, an NR antagonist, combined with $2 \mathrm{~Hz}$ EA at the Zusanli and Sanyinjiao acupoints could strengthen the analgesic effect during thermal hypersensitivity of the hindpaw in a pain model after injection with complete Freund's adjuvant [34]. These findings indicate that NMDA are potential targets for acupuncture. Recently, yamamoto et al. reported that mechanoreceptor blocker gadolinium significantly attenuated the MA- and EA-induced physiological bradycardic response and depressor response. They suggest that the mechanoreceptors are involved in the sensory mechanisms for both MA and EA. Wu et al. suggest that TRPV1 is highly expressed in different anatomical layers of ST36 acupoint. They show that TRPV1 was not only existed in nerve fibers but also expressed in skeletal muscle cells and fibroblasts. Injection of TRPV1 agonists into ST36 showed that replicated acupuncture analgesia [10].

\section{Conclusion}

We conclude that MA and EA share the same signal transmission pathway. All of our manipulations significantly elicited the TRPV1-associated signaling pathway, pPKA, pPI3K, pPKC-pERK, and pAKT, as well as activating pNR1-pCaMKII $\alpha$ excitatory signaling transduction in the peripheral DRG and central SC-SSC of mice. We also showed that pp38, pJNK, pNR2B, pCaMKII $\delta$, and pCaMKII $\gamma$ were unaltered after the MA and EA manipulations. Our results provide strong evidence because these mechanisms were abolished in TRPV1-/mice. These results suggest that EA manipulation mechanically activated the peripheral and central nervous systems through a TRPV1-associated signaling pathway. We suggest that the TRPV1 signaling pathway is highly correlated to clinical effect of Acupuncture. 


\section{Cellular Physiology Cell Physiol Biochem 2018;49:1813-1824 \begin{tabular}{ll|l} 
and Biochemistry Published online: 20 September, 2018 & $\begin{array}{l}\text { C } 2018 \text { The Author(s). Published by S. Karger AG, Basel } \\
\text { www.karger.com/cpb }\end{array}$ \\
\hline
\end{tabular}}

\section{Acknowledgements}

This work was financially supported by the "Chinese Medicine Research Center, China Medical University" from The Featured Areas Research Center Program within the framework of the Higher Education Sprout Project by the Ministry of Education (MOE) in Taiwan and MOST 107-2320-B-039-033.

C.H.C. and L.Y.W. conceived the study design. C.H.C. and C.M.Y employed the experiments, collected, and analyzed the data. H.C.L., H.H.C., and L.Y.W. wrote the manuscript. L.Y.W. obtained the research grants for the current study. All the authors reviewed the manuscript and agreed for submission.

\section{Disclosure Statement}

The authors declare that there are no conflicts of interest.

\section{References}

1 Abraham TS, Chen ML, Ma SX: TRPV1 expression in acupuncture points: response to electroacupuncture stimulation. J Chem Neuroanat 2011;41:129-136.

$\longrightarrow 2$ Ataei N, Sabzghabaee AM, Movahedian A: Calcium/Calmodulin-dependent Protein Kinase II is a Ubiquitous Molecule in Human Long-term Memory Synaptic Plasticity: A Systematic Review. Int J Prev Med 2015;6:88.

-3 Barnes JM, Henley JM: Molecular characteristics of excitatory amino acid receptors. Prog Neurobiol 1992;39:113-133.

4 Caterina MJ, Schumacher MA, Tominaga M, Rosen TA, Levine JD, Julius D: The capsaicin receptor: a heatactivated ion channel in the pain pathway. Nature 1997;389:816-824.

5 Chen CY, Lin CN, Chern RS, Tsai YC, Chang YH, Chien CH: Neuronal Activity Stimulated by Liquid Substrates Injection at Zusanli (ST36) Acupoint: The Possible Mechanism of Aquapuncture. Evid Based Complement Alternat Med 2014;2014:627342.

-6 Choi SI, Lim JY, Yoo S, Kim H, Hwang SW: Emerging Role of Spinal Cord TRPV1 in Pain Exacerbation. Neural Plast 2016;2016:5954890.

7 Choowanthanapakorn M, Lu KW, Yang J, Hsieh CL, Lin YW: Targeting TRPV1 for Body Weight Control using TRPV1(-/-) Mice and Electroacupuncture. Sci Rep 2015;5:17366.

8 Denda M, Tsutsumi M: Possible role of epidermal keratinocytes in the construction of acupuncture meridians. J Acupunct Meridian Stud 2014;7:92-94.

-9 Egbuniwe 0, Grover S, Duggal AK, Mavroudis A, Yazdi M, Renton T, Di Silvio L, Grant AD: TRPA1 and TRPV4 activation in human odontoblasts stimulates ATP release. J Dent Res 2014;93:911-917.

-10 Ferrini F, Salio C, Vergnano AM, Merighi A: Vanilloid receptor-1 (TRPV1)-dependent activation of inhibitory neurotransmission in spinal substantia gelatinosa neurons of mouse. Pain 2007;129:195-209.

11 Goldman N, Chen M, Fujita T, Xu Q, Peng W, Liu W, Jensen TK, Pei Y, Wang F, Han X, Chen JF, Schnermann J, Takano T, Bekar L, Tieu K, Nedergaard M: Adenosine A1 receptors mediate local anti-nociceptive effects of acupuncture. Nat Neurosci 2010;13:883-888.

12 Han JS: Acupuncture: neuropeptide release produced by electrical stimulation of different frequencies. Trends Neurosci 2003;26:17-22.

13 Hollmann M, Heinemann S: Cloned glutamate receptors. Annu Rev Neurosci 1994;17:31-108.

14 Huang CP, Chen HN, Su HL, Hsieh CL, Chen WH, Lai ZR, Lin YW: Electroacupuncture Reduces Carrageenanand CFA-Induced Inflammatory Pain Accompanied by Changing the Expression of Nav1.7 and Nav1.8, rather than Nav1.9, in Mice Dorsal Root Ganglia. Evid Based Complement Alternat Med 2013;2013:312184.

15 Jang JH, Nam TS, Jun J, Jung SJ, Kim DW, Leem JW: Peripheral NMDA Receptors Mediate Antidromic Nerve Stimulation-Induced Tactile Hypersensitivity in the Rat. Mediators Inflamm 2015;2015:793624.

16 Jang JY, Kim HN, Koo ST, Shin HK, Choe ES, Choi BT: Synergistic antinociceptive effects of N-methyl-Daspartate receptor antagonist and electroacupuncture in the complete Freund's adjuvant-induced pain model. Int J Mol Med 2011;28:669-675. 


\section{Cellular Physiology Cell Physiol Biochem 2018:49:1813-1824 and Biochemistry DOI: 10.1159/000493627 2018 O 2018 The Author(s). Published by S. Karger AG, Basel

17 Kuo CT, Lin YW, Tang NY, Cheng CY, Hsieh CL: Electric stimulation of the ears ameliorated learning and memory impairment in rats with cerebral ischemia-reperfusion injury. Sci Rep 2016;6:20381.

18 Lin JG, Hsieh CL, Lin YW: Analgesic Effect of Electroacupuncture in a Mouse Fibromyalgia Model: Roles of TRPV1, TRPV4, and pERK. PLoS One 2015;10:e0128037.

-19 Lin YW, Hsieh CL: Electroacupuncture at Baihui acupoint (GV20) reverses behavior deficit and long-term potentiation through $\mathrm{N}$-methyl-d-aspartate and transient receptor potential vanilloid subtype 1 receptors in middle cerebral artery occlusion rats. J Integr Neurosci 2010;9:269-282.

-20 Lin YW, Hsieh CL: Auricular electroacupuncture reduced inflammation-related epilepsy accompanied by altered TRPA1, pPKCalpha, pPKCepsilon, and pERk1/2 signaling pathways in kainic acid-treated rats. Mediators Inflamm 2014;2014:493480.

21 Lu KW, Hsu CK, Hsieh CL, Yang J, Lin YW: Probing the Effects and Mechanisms of Electroacupuncture at Ipsilateral or Contralateral ST36-ST37 Acupoints on CFA-induced Inflammatory Pain. Sci Rep 2016;6:22123.

22 Lynch JW: Molecular structure and function of the glycine receptor chloride channel. Physiol Rev 2004;84:1051-1095.

23 Ma C, Feng KH, Yan LP: [Effects of electroacupuncture on long-term potentiation of synaptic transmission in spinal dorsal horn in rats with neuropathic pain]. Zhen Ci Yan Jiu 2009;34:324-328.

24 Malcangio M, Bowery NG: GABA and its receptors in the spinal cord. Trends Pharmacol Sci 1996;17:457462.

25 Nagy GG, Watanabe M, Fukaya M, Todd AJ: Synaptic distribution of the NR1, NR2A and NR2B subunits of the N-methyl-d-aspartate receptor in the rat lumbar spinal cord revealed with an antigen-unmasking technique. Eur J Neurosci 2004;20:3301-3312.

-26 Okamoto K, Bosch M, Hayashi Y: The roles of CaMKII and F-actin in the structural plasticity of dendritic spines: a potential molecular identity of a synaptic tag? Physiology 2009;24:357-366.

27 Ryu JW, Lee JH, Choi YH, Lee YT, Choi BT: Effects of protein phosphatase inhibitors on the phosphorylation of spinal cord N-methyl-D-aspartate receptors following electroacupuncture stimulation in rats. Brain Res Bull 2008;75:687-691.

28 Wang JY, Li H, Zhang L, Ma CM, Wang JL, Lai XS, Zhou SF: Adenosine as a probing tool for the mechanistic study of acupuncture treatment. Clin Exp Pharmacol Physiol 2014;41:933-939.

29 Wong H, Dong XD, Cairns BE: Nerve growth factor alters the sensitivity of rat masseter muscle mechanoreceptors to NMDA receptor activation. J Neurophysiol 2014;112:2275-2282.

-30 Wu L, Oshima T, Shan J, Sei H, Tomita T, Ohda Y, Fukui H, Watari J, Miwa H: PAR-2 activation enhances weak acid-induced ATP release through TRPV1 and ASIC sensitization in human esophageal epithelial cells. Am J Physiol Gastrointest Liver Physiol 2015;309:G695-702.

-31 Wu SY, Chen WH, Hsieh CL, Lin YW: Abundant expression and functional participation of TRPV1 at Zusanli acupoint (ST36) in mice: mechanosensitive TRPV1 as an "acupuncture-responding channel". BMC Complement Altern Med 2014;14:96.

32 Ye TS, Du ZH, Li ZH, Xie WX, Huang KT, Chen Y, Chen ZY, Hu H, Wang JL, Fang JQ: Repeated Electroacupuncture Persistently Elevates Adenosine and Ameliorates Collagen-Induced Arthritis in Rats. Evid Based Complement Alternat Med 2016;2016:3632168.

-33 Yung KK: Localization of glutamate receptors in dorsal horn of rat spinal cord. Neuroreport 1998;9:16391644.

34 Zhao ZQ: Neural mechanism underlying acupuncture analgesia. Prog Neurobiol 2008;85:355-375. 